10

\title{
K вопросу о трехкратной брэгговской дифракции в кристалле парателлурита
}

\author{
() В.М. Котов, С.В. Аверин, А.И. Воронко, Е.В. Котов, С.А. Тихомиров
}

Институт радиотехники и электроники им. В.А. Котельникова РАН, 141120 Фрязино, Московская обл., Россия

e-mail: vmk277@ire216.msk.su

(Поступило в Редакцию 23 августа 2016 г.)

Исследован вариант трехкратной брэгговской дифракции в кристалле парателлурита, когда плоскость дифракции наклонена к оптической оси кристалла. Показано, что эффективные фотоупругие константы для анизотропной и изотропной дифракций практически не меняются с изменением угла наклона плоскости дифракции. Экспериментально получено трехкратное брэгговское рассеяние оптического излучения с длиной волны $0.63 \mu \mathrm{m}$ в парателлурите на „медленной“ акустической волне с частотой $47.3 \mathrm{MHz}$. При подводимой к пьезопреобразователю электрической мощности $0.69 \mathrm{~W}$ относительные эффективности дифракционных порядков составили $\sim 0.4,0.4,0.1$ и 0.1 соответственно.

DOI: 10.21883/JTF.2017.07.44682.2021

\section{Введение}

Одной из интересных особенностей акустооптической (AО) дифракции в анизотропных средах является возможность двух- и трехкратного брэгговского рассеяний [1-3], когда оптическое излучение дифрагирует в два или три дифракционных порядка, каждый из которых является брэгговским. Выполнение строгого брэгговского синхронизма позволяет сконцентрировать все оптическое излучение в наивысшем порядке, при этом угол отклонения и изменение частоты света равны соответственно $n \Theta_{p}$ и $n f$, где $\Theta_{p}$ и $f-$ угол рассеяния и частота смещения одиночного акта дифракции, $n-$ номер дифракционного порядка. Интерес к многократной брэгговской дифракции возрос в настоящее время в связи с обработкой оптических изображений. Выяснилось, что многократные акты рассеяния позволяют получать двумерный контур изображения в процессе его фурьеобработки [4-6]. Поэтому более детальные исследования многократных процессов брэгговского рассеяния расширяют возможности использования $\mathrm{AO}$ взаимодействия для задач обработки оптических изображений.

В работе [2] достаточно подробно рассмотрено это явление на примере многократной (многофононной) дифракции в парателлурите $\left(\mathrm{TeO}_{2}\right)$, наиболее известном и широко используемом на практике АО кристалле. Самая эффективная дифракция в $\mathrm{TeO}_{2}$ происходит при рассеянии света на поперечной звуковой волне $V_{s}$, распространяющейся вдоль направления кристалла с направлением сдвига вдоль [1ㅣ] [2,7,8]. При этом возможна только анизотропная дифракция, сопровождающаяся „перебросом“ волнового вектора света с одной волновой поверхности на другую. Изотропная же дифракция, происходящая в пределах одной волновой поверхности, невозможна, для нее соответствующая фотоупругая константа равна нулю $[2,7,8]$. В связи с этим в [2] рассматривались варианты многофононной дифракции в $\mathrm{TeO}_{2}$, использующие только анизотропную дифракцию. В этом случае многофононная дифракция возможна только с расстройкой брэгговского синхронизма, при этом наименьшая расстройка обеспечивается только в случае, когда плоскость дифракции содержит оптическую ось кристалла. Здесь дифракция происходит на фиксированной звуковой частоте. Например, трехфононная дифракция оптического излучения с длиной волны $0.63 \mu \mathrm{m}$ происходит на частоте звука, равной $\sim 27 \mathrm{MHz}$.

В настоящей работе рассматривается трехкратное брэгговское рассеяние света, когда плоскость дифракции наклонена к оптической оси кристалла. При достаточно больших углах наклона гиротропию кристалла можно не учитывать, задача существенно упрощается. В работе выполнен полный расчет процесса дифракции, получены его характеристики. В частности, выяснилось, что частота звука для реализации трехкратной дифракции не фиксирована, она меняется линейно с ростом угла наклона; становится возможной реализация изотропной дифракции на звуковой волне $V_{s}$; ряд параметров дифракции практически не зависит от угла наклона и т.п. Все это существенно расширяет возможности акустооптики для управления параметрами оптического излучения посредством многофононного брэгговского рассеяния.

\section{1. Теория}

На рис. 1 представлена трехмерная векторная диаграмма трехкратной АО дифракции в одноосном кристалле, плоскость дифракции $P$ наклонена к оптической оси кристалла $O Z$ на угол $\alpha$. Для определенности рассматривается одноосный положительный кристалл. Падающее излучение с волновым вектором $\mathbf{K}_{0}$ в результате АО взаимодействия с акустической волной q 


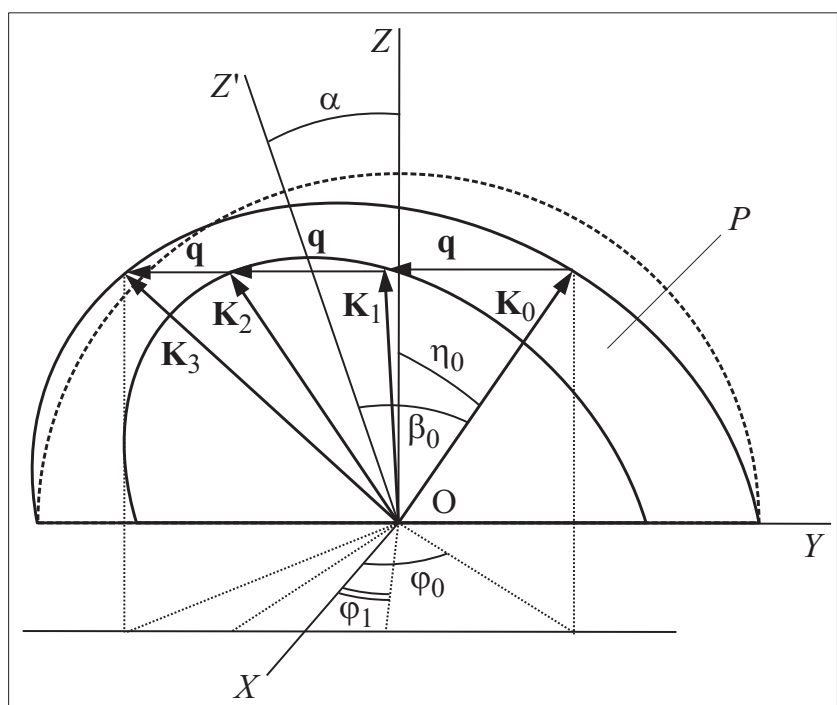

Pис. 1. Векторная диаграмма трехкратной брэгговской дифракции.

$(\mathbf{q} \perp O Z)$, последовательно дифрагирует в направления $\mathbf{K}_{1}, \mathbf{K}_{2}$ и $\mathbf{K}_{3}$, являющиеся первым, вторым и третьим дифракционными порядками соответственно. Ось $O Z^{\prime}$ является проекцией оптической оси $O Z$ на плоскость дифракции $P$. Угол между $\mathbf{K}_{0}$ и $O Z^{\prime}$ обозначен $\beta_{0}$. Будет подразумеваться, что углы между векторами $\mathbf{K}_{1}, \mathbf{K}_{2}, \mathbf{K}_{3}$ и $O Z^{\prime}$ равны $\beta_{1}, \beta_{2}, \beta_{3}$. Последние углы на диаграмме не показаны, чтобы не перегружать рисунок. Углы между векторами $\mathbf{K}_{0}-\mathbf{K}_{3}$ и оптической осью $O Z$ равны $\eta_{0}-\eta_{3}$, показан только угол $\eta_{0}$. Углы между проекциями $\mathbf{K}_{0}-\mathbf{K}_{3}$ на плоскость $O X Y$ и осью $O X$ равны $\varphi_{0}-\varphi_{3}$, приведены только углы $\varphi_{0}$ и $\varphi_{1}$ по вышеуказанным соображениям. Полагается, что дифракция происходит в монокристалле $\mathrm{TeO}_{2}$. Будем рассматривать дифракцию излучения с длиной волны $0.63 \mu \mathrm{m}$. Оси $O X, O Y, O Z$ соответствуют кристаллографическим направлениям [110], [110] и [001] кристалла $\mathrm{TeO}_{2}$. Полагаем, что угол наклона $\alpha$ больше $3^{\circ}$, поэтому гиротропией кристалла для выбранной длины волны света пренебрегаем. В этом приближении поверхности волновых векторов одноосного кристалла описываются выражениями $[8,9]$

$$
\frac{k_{z}^{2}}{k_{n}^{2}}+\frac{k_{y}^{2}}{k_{e}^{2}}=1, \quad k_{z}^{2}+k_{y}^{2}=k_{0}^{2}
$$

где $k_{0}=2 \pi n_{0} / \lambda k_{e}=2 \pi n_{e} / \lambda, k_{n}=2 \pi n_{\alpha} / \lambda$,

$$
n_{\alpha}=n_{0} n_{e}\left(n_{0}^{2} \sin ^{2} \alpha+n_{e}^{2} \cos ^{2} \alpha\right)^{-1} .
$$

Здесь $n_{0}, n_{e}$ - главные показатели преломления кристалла, $\lambda$ - длина волны света, $\alpha-$ угол наклона плоскости дифракции к оптической оси $O Z, k_{z}, k_{y}-$ проекции волнового вектора света на оси $O Z^{\prime}$ и $O Y$ соответственно. Нетрудно показать, что волновой вектор звука трехкратной брэгговской дифракции определяется соотношением

$$
q=2 k_{e} \sqrt{\frac{k_{n}^{2}-k_{0}^{2}}{9 k_{n}^{2}-k_{e}^{2}}}
$$

Все последующие вычисления выполнялись на основании выражений (1), (2) и векторной диаграммы при следующих параметрах: $\lambda=0.63 \mu \mathrm{m}, n_{0}=2.26, n_{e}=2.41$. Скорость звука в кристалле бралась равной $617 \mathrm{~m} / \mathrm{s}$. $\mathrm{B}$ силу симметрии $\mathrm{AO}$ взаимодействия $\beta_{0}=\beta_{3}$ и $\beta_{1}=\beta_{2}$. Расчеты показали, что частота звука растет линейно с ростом $\alpha$ и подчиняется соотношению $f \approx 10^{7} \alpha$, где частота $f$ выражается в $\mathrm{Hz}$, угол $\alpha-$ в deg. Например, при $\alpha=5^{\circ}$ частота равна $50 \mathrm{MHz}$. Углы $\beta_{0}-\beta_{3}$ также линейно зависят от $\alpha$, они описываются соотношениями $\beta_{0}=\beta_{3} \approx 0.4 \alpha, \beta_{1}=\beta_{2} \approx 0.12 \alpha$. Углы $\eta_{0}-\eta_{3}$ и $\varphi_{0}-\varphi_{3}$ связаны с углами $\alpha$ и $\beta_{0}-\beta_{3}$ соотношениями

$$
\begin{gathered}
\cos \eta_{0-3}=\cos \alpha \cos \beta_{0-3}, \\
\cos \varphi_{0-3}=\sin \alpha \cos \beta_{0-3} / \sin \eta_{0-3} .
\end{gathered}
$$

Знание углов $\eta_{0}-\eta_{3}$ и $\varphi_{0}-\varphi_{3}$ необходимо для вычисления фотоупругих констант. Здесь также в силу симметрии рассматриваемого варианта дифракции $\eta_{0}=\eta_{3}$, $\eta_{1}=\eta_{2}, \varphi_{0}=\varphi_{3}, \varphi_{1}=\varphi_{2}$. Расчеты показывают, что $\varphi_{0}=\varphi_{3}=21.6^{\circ}, \varphi_{1}=\varphi_{2}=7.52^{\circ}$, и эти значения не меняются с изменением угла $\alpha(!)$. Эффективные фотоупругие константы для дифракции „необыкновенного“ луча в „обыкновенный“ $(e-o)$ и „обыкновенного“ в „обыкновенный“ $(o-o)$ вычислялись согласно выражениям

$$
\begin{aligned}
& P_{\mathrm{eff}(e-o)}==0.5\left(P_{11}-P_{12}\right) \cos \eta_{0} \cos \left(\varphi_{0}+\varphi_{1}\right), \\
& P_{\mathrm{eff}(o-o)}=-0.5\left(P_{11}-P_{12}\right) \sin 2 \varphi_{1} .
\end{aligned}
$$

Оказалось, что $P_{\operatorname{eff}(e-o)}$ и $P_{\operatorname{eff}(o-o)}$ практически не зависят от $\alpha$, они равняются 0.105 и 0.032 соответственно.

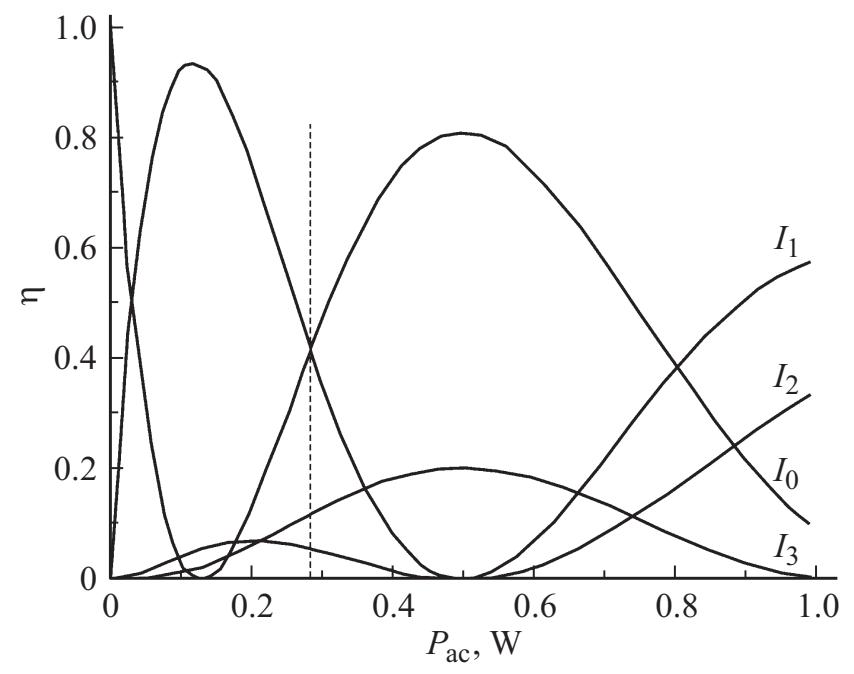

Рис. 2. Зависимости эффективности дифракции 0-, 1-, 2- и 3-го брэгговских порядков от акустической мощности $P_{a c}$. 
Обе фотоупругие константы не равны нулю. Другими словами, расчеты указывают на возможность существования трехкратного рассеяния с использованием как анизотропного, так и изотропного вариантов дифракции.

За основу расчета эффективностей дифракционных порядков трехкратной брэгговской дифракции использована методика, изложенная в $[1,8]$. Параметры Рамана-Ната $v_{e-o}$ и $v_{o-o}$ для анизотропного и изотропного актов дифракции, входящие в уравнения, вычислялись согласно выражениям

$\nu_{e-o}=\frac{2 \pi}{\lambda} \sqrt{\frac{M_{2(e-o)} L}{2 H} P_{a c}}, \quad v_{o-o}=\frac{2 \pi}{\lambda} \sqrt{\frac{M_{2(o-o)} L}{2 H} P_{a c}}$,

где $L$ и $H$ - длина АО взаимодействия и высота акустического столба соответственно, $M_{2(e-o)}$ и $M_{2(o-o)}$ - коэффициенты АО качества материала, равные $M_{2(e-o)}=\frac{n_{0}^{6} P_{\text {eff }(l-o)}^{2}}{\rho V^{3}}, \quad M_{2(o-o)}=\frac{n_{0}^{6} P_{\text {eff }(o-o)}^{2}}{\rho V^{3}}$, где $\rho-$ плотность кристалла, $V-$ скорость звуковой волны. В нашем случае $M_{2(e-o)} \approx 1040 \cdot 10^{-18} \mathrm{~s}^{3} / \mathrm{g}, M_{2(o-o)} \approx$ $\approx 92 \cdot 10^{-18} \mathrm{~s}^{3} / \mathrm{g}$. В выражении (6) $P_{a c}-$ мощность звука.

На рис. 2 приведены зависимости эффективности дифракции $\eta$ нулевого, 1-, 2- и 3-го дифракционных порядков, обозначенные как $I_{0}, I_{1}, I_{2}$ и $I_{3}$, от мощности звука $P_{a c}$. Видно, что характер изменения эффективности дифракции всех порядков - осциллирующий. Вертикальной штриховой линией указана мощность звука и соответствующие ей интенсивности дифракционных порядков, которые наблюдались нами в эксперименте (см. ниже).

Отметим попутно, что, согласно расчетам, все излучение может быть сконцентрировано в третьем порядке дифракции. Это реализуется при мощности звука $P_{a c} \approx 4.8 \mathrm{~W}$. Таким образом, существует возможность практически $100 \%$-ного отклонения всего падающего излучения в наивысший порядок.

\section{2. Эксперимент и обсуждение экспериментальных результатов}

На рис. 3 приведена оптическая схема экспериментальной установки. Оптическое излучение с длиной волны $0.63 \mu \mathrm{m}$, генерируемое $\mathrm{He}-\mathrm{Ne}$-лазером 1 , направляется на четверь-волновую пластинку 2 , после которой на $\mathrm{AO}$ ячейку 3. На ячейку подается электрический сигнал 4. За ячейкой расположен анализатор 5 для анализа поляризаций выходящих из ячейки лучей. Пятна от лучей наблюдаются на экране 6 . Пятно $7-$ непродифрагировавший луч, пятна 8,9 и $10-$ лучи, продифрагировавшие в первый, второй и третий порядки соответственно. АО ячейка 3 выполнена из монокристалла $\mathrm{TeO}_{2}$ с размерами $1.0 \times 1.0 \times 1.0 \mathrm{~cm}$ вдоль направлений [001], $[110]$ и $[1 \overline{1} 0]$ кристалла. Вдоль $[1 \overline{1} 0]$ распространялась „медленная“ звуковая волна, генерируемая пьезопреобразователем из $\mathrm{LiNbO}_{3}$. Частота звука $-47.3 \mathrm{MHz}$.

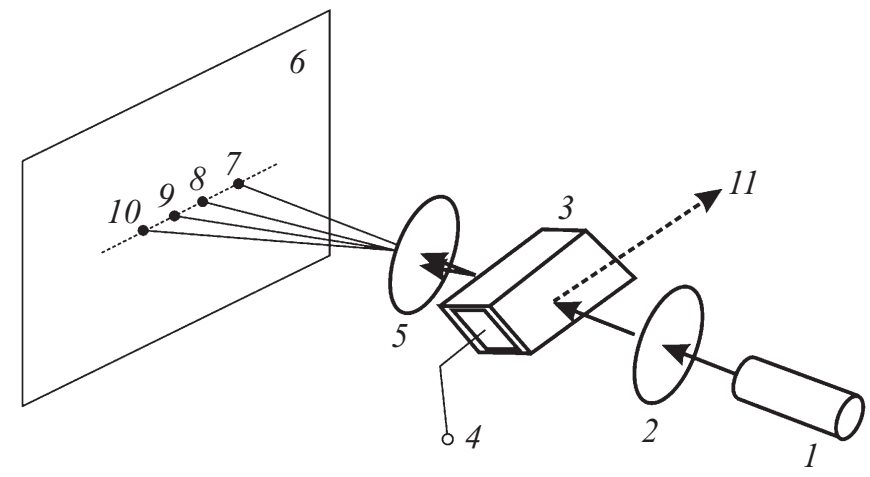

Рис. 3. Оптическая схема экспериментальной установки.

Длина $\mathrm{AO}$ взаимодействия $-0.6 \mathrm{~cm}$. Путем наклона $\mathrm{AO}$ ячейки добивалась ситуация, когда появлялись все дифрагировавшие лучи с максимально возможной эффективностью. При наклоне плоскости дифракции на угол $\sim 48^{\circ}$, определяемом по углу отражения луча 11 от передней грани кристалла с соответствующим пересчетом для учета преломления на границе и напряжении на пьеэопреобразователе $8.3 \mathrm{~V}$, относительные эффективности лучей составили $\sim 0.4,0.4,0.1$ и 0.1 для $0-, 1$, 2- и 3-го порядков соответственно. Электрическая мощность, соответствующая напряжению $8.3 \mathrm{~V}$ при нагрузке $50 \Omega$, равна $0.69 \mathrm{~W}$. Полученные эффективности соответствуют случаю, отмеченному на рис. 3 вертикальной линией, соответствующей $P_{a c} \approx 0.28 \mathrm{~W}$. Это означает, что коэффициент преобразования электрической мощности в акустическую равен $\sim 0.41$. Все расхождения между теоретическими и экспериментальными данными могут быть объяснены неполным согласованием импедансов электрического и оптического трактов, неточностью используемой нами модели, конечной расходимостью оптических и акустических лучей, неточной ориентацией кристалла и т.п. В любом случае наблюдается хорошее согласие теории и эксперимента.

\section{3. Заключение}

На основании изложенного можно сделать следующие выводы.

1. Рассмотрена трехкратная брэгговская дифракция в плоскости, наклоненной к оптической оси кристалла на угол $\alpha$. Показано, что рассматриваемая геометрия позволяет рассчитать все параметры взаимодействия: частотно-угловые характеристики, эффективные фотоупругие константы, эффективность дифракции всех порядков как функции мощности звука.

2. Показано, что эффективные фотоупругие константы, определяющие эффективность трехкратной дифракции, не зависят от наклона плоскости дифракции, и на длине волны света $0.63 \mu \mathrm{m}$ равны 0.105 и 0.0312 для анизотропного и изотропного актов рассеяния соответственно. 
3. Эксперименты, выполненные на основе монокристалла $\mathrm{TeO}_{2}$, выбранного в качестве материала $\mathrm{AO}$ ячейки, подтвердили основные выводы теории: получена трехкратная брэгговская дифракция на частоте звука $47.3 \mathrm{MHz}$ с относительными эффективностями, равными $0.4,0.4,0.1$ и 0.1 для 0-, 1-, 2- и 3-го дифракционных порядков соответственно.

Полученные результаты могут найти применение в системах обработки оптических сигналов, в которых используется многократное брэгговское рассеяние.

Работа выполнена при частичной финансовой поддержке РФФИ (гранты № 16-07-00064, 14-07-00014) и гранта президента Российской Федерации для государственной поддержки ведущих научных школ Российской Федерации НШ-3317.2010.9.

\section{Список литературы}

[1] Балакший В.И., Парыгин В.Н., Чирков Л.Е. Физические основы акустооптики. М.: Радио и связь, 1985. 280 с.

[2] Раковский В.Ю., Щербаков А.С. // ЖТФ. 1990. Т. 60. Вып. 7. C. $107-114$.

[3] Волошинов В.Б., Парыгин В.Н., Чирков Л.Е. Некоторые особенности анизотропной дифракции Брэгга // Вестн. МГУ. Сер. 3. Физ., астр. 1976. Т. 17. № 3. С. 305-312.

[4] Котов В.М., Аверин С.В., Шкердин Г.Н., Воронко А.И. // Квант. электрон. 2010. Т. 40. Вып. 4. С. 368-370.

[5] Котов В.М., Шкердин Г.Н., Булюк А.Н. // Квант. электрон. 2011. T. 41. Вып. 12. С. 1109-1113.

[6] Котов В.М., Шкердин Г.Н., Григорьевский В.И. // Радиотехн. и электрон. 2013. Т. 58. Вып. 3. С. 256-263.

[7] Xu J., Stroud R. Acousto-Optic Devices: Principles, Design and Applications. John Wiley and Sons, Inc., 1992. 652 p.

[8] Котов В.М. Акустооптика. Брэгговская дифракция многоцветного излучения. М.: Янус-К, 2016. 286 с.

[9] Борн М., Вольф Э. Основы оптики. М.: Наука, 1973. 719 с. 